\title{
Structural domain determination — practicality and pitfalls
}

\author{
JI Mathis Zostrich Geotechnical, USA
}

\begin{abstract}
The concept of a geotechnical structural domain is nebulous, depending in part upon the requirements of the project, the data available and the experience of the involved geotechnical engineer(s).

However, such geotechnical structural domains are necessary for accurate, and comprehensive, engineering design of openings in rock. As such, geologic structural systems affecting the area in question must be evaluated and quantified. This may require multiple iterations of data collection. However many iterations that are required, the evaluation, and projection, of the data must be conducted such that it accommodates the limitations of the data collection methodologies. Without this interaction and understanding of shortfalls in data collection methodology adequacy, the defined domains may not only be spatially, but also descriptively, in error.

The geotechnical structural domains defined are constrained by practicality as well. While one can spend an exorbitant amount of time, and money, on describing a large number of geotechnical structural domains, it may be questioned if this is realistic or necessary for the project at hand. At times, seemingly insignificant differences may be of substantial import to a project. Alternatively, multiple large geotechnical structural domains with obvious differences may have little impact on a specific project given, for example, the orientation of the opening, or the excavation methodology chosen.
\end{abstract}

Structural geologic understanding, knowledge of data collection and evaluation biases and shortfalls, and understanding of the impact of what factors may be critical to design are all necessary when defining and describing useful geotechnical structural domains.

\section{Introduction}

A geotechnical structural domain (hereafter termed a structural domain) is here defined as a volume of rock having geotechnically similar discontinuity spatial, and specific associated structural, characteristics. 'Spatial characteristics' refer to orientation, persistence and spacing (density), together with their associated distributions. At times, infill mineralogy, local structural alteration and roughness may be included, as well as any other structurally controlled parameters that distinguish the area in a significant design sense. This differs from a geotechnical domain in that geotechnically significant factors may be specifically excluded from a structural domain as they are not structurally controlled.

For example, if the structural characteristics and distributions are similar in a fresh versus altered intrusive but the intact compressive strengths are inherently (and substantially) different then this could be considered a single structural domain but would comprise two geotechnical domains.

Alternatively, if the persistence of a critical discontinuity set was substantially greater in one portion of a uniform lithologic host, and the intact compressive strengths were near identical, then two structural domains would exist and define two geotechnical domains.

Thus, each structural domain will normally be a geotechnical domain, but not every geotechnical domain is a unique structural domain. This is especially true for geotechnical domains delineated by rock mass ratings. While there may be structural differences that result in varying rock mass ratings, these are not generally structural domains unless the controlling structural features are specifically identified and described. 
It would be difficult to ascribe a hard and fast rule to structural domain determination. One could theoretically construct a 'pure' structural domain; however, the ridiculous extreme of this would be each block of rock would be its own structural domain. The other extreme is simply combining all the structural characteristics of an area and defining the entire area, or lithologic unit, as a single structural domain. It is obvious that a huge range exists between these two extremes - a range influenced by the amount and type of geologic structural data collected, the data collection methodologies employed, the analytic methods utilised to reduce the data, and the end purpose of the defined structural domains. Neither of the above end members of structural domain determination is, in most cases, practically applicable to accurate engineering design.

In order to properly determine 'appropriate' structural domains for engineering design, one must define and understand the local major structure, the genesis and relation of the structures (if possible), the lithologic hosts and alteration (again, if possible), the rock fabric incorporated in the faulted and altered lithologic units and finally, relate all this to opening design.

\section{Data collection}

As a structural domain is defined by natural discontinuities in the rock mass, sampling of the spatial characteristics of discontinuities is required to first, define the characteristics of the populations and second, provide the ability to segregate the population by area; both for the purposes of engineering design.

There are at least two major size related groupings of discontinuities that must be considered. These are: 1) rock fabric, or the innumerable small scale discontinuities in the rock mass, commonly referred to as joints, and 2) major structure (faults, shears, intermediate faults etc.).

Data collection must be designed such as to adequately collect the requisite information regarding both scales of discontinuities. This is not a trivial task and one that appears to escape many practitioners.

\subsection{Methods and method bias}

\subsubsection{Excavation and/or outcrop information}

Surface mapping both on natural slopes and man-made excavations (including tunnels, stopes etc.) provides potentially the best information for discontinuity characterisation and generally allows one to obtain a good estimate of discontinuity persistence. Areal traverse mapping and/or photogrammetric mapping (aerial or terrestrial), depending on how they are conducted, can provide good to very good discontinuity population information (Mathis 2015). LIDAR, as well as geophysical methods, can also be quite useful for determination of major structure location (and possibly orientation) acting as potential (first cut) domain bounds.

Scan line mapping is not recommended, either physically or photogrammetrically. The scanline method is tremendously susceptible to bias. While similar to a drillhole in nature, it varies in that the mapper can, and often does, go outside the acceptance criteria (e.g. linearity assumption, planarity, persistence censoring and truncation, number of samples etc.) to map structures. Not only does it contain a multitude of inherent biases, including a difficult if not impossible to rectify orientation bias, but the collected data is extremely difficult to reduce, at least in terms of structural persistence.

In contrast to line mapping, cell, or window, mapping, is an area mapping technique easily implemented with photogrammetric or scanning techniques. For cell mapping, a window of a pre-determined shape and dimensions is defined on the mapping face. All features greater than a pre-determined minimum length found within the window are mapped as to orientation, trace length, truncation bounds relative to the window etc. As this is an area technique, it is recommended over the scanline technique as it is less sensitive to orientation bias. 


\subsubsection{Drill hole information}

Subsurface information (tunnels, stopes etc. excluded) can, unfortunately, generally only be collected from drillhole information. Additionally, while drillhole information can provide adequate (although heavily orientation biased) information regarding discontinuity orientations and density, it struggles (some would say cannot) with providing information regarding persistence.

Given the biases associated with drill information, considerable thought must be given to the drillhole discontinuity orientation methods employed. Acoustic televiewer techniques (ATV) can be utilised in drillholes that will maintain a fluid column. This method provides relatively accurate orientation information (given, of course, the skill of the instrument operator and the logger) even through heavily fractured zones. Barrel orientation techniques generally do not provide information in heavily broken zones where orientation is lost. This is a critical flaw of the technique and one which precludes recommendation of the method by this author (Mathis 2015). It may be noted that the author, with over 20 years of experience utilising the ATV technique, has yet to encounter a fracture zone that could not be imaged with an ATV given proper mud and drilling techniques.

Given that a drillhole is essentially a line in space, careful planning of the drillhole orientation and location is required to maximise the relevant data collected (i.e. to pierce the appropriate major structure and/or discontinuity sets) and to minimise the relevant information lost. In many cases this requires multiple holes in varying orientations.

Some methods of discontinuity data collection obtain only geometric discontinuity spatial characteristics, (i.e. photogrammetry, ATV drillhole logging). This leaves an information gap regarding discontinuity surface conditions (roughness, infill thickness, infill minerals). This lack of information can, in most cases, only be addressed by physical mapping of either outcrop (or any exposed rock face) or drill core.

\section{$2.2 \quad$ Location bias}

Biases exist not only in the methodologies employed, as discussed briefly in the section above, but in terms of sample locations. As such, an area that is to be described in terms of structural domains must be sampled in a fashion which minimises location biases. Some examples of location bias are as follow:

- Consider, for example, a typical shear or vein hosted deposit. The drillhole information collected from the deposit will generally be directed towards defining reserves. In many cases the drillholes are oriented along strike or cross sectionally through the mineralised zone. These drillholes provide biased information as to location, given they will be primarily located in a highly altered and/or broken environment, as well as orientation. If the mineralised zone is to be mined by open pit methods, there is little, if any, discontinuity information available from the pit walls.

- In a similar vein, consider the mapping that may be conducted in a newly excavated, or alternatively, a historic pit excavation. Near surface mapping will be biased due to weathering. In some cases areas simply cannot be mapped due to alteration/overburden concerns. In the case of an historic pit slope, blast damage and weathering may contribute to inaccurate values for discontinuity spatial characteristics both locally in situ and at depth.

- Lithologies can locally influence structure, and as such, must be considered when collecting data, as contact effects can considerably influence the local structural data. Additionally, one must consider what in what units the excavation will be hosted. It was observed in one case where detailed mapping was conducted in metasediments on an existing pit wall. The design slope in the area was, however, to be excavated in an intrusive located behind the existing pit slope. Not only was the collected data not applicable to the design slope but unnecessary costs were incurred in the collection of this data.

- Core hole locations can be unnecessarily 'fixed' in space and orientation based upon design assumptions. For example, it has been observed on multiple occasions that drillholes were drilled 
parallel, but just behind, the assumed design pit wall. These drillholes exhibit generally extreme orientation bias with regards to most adverse structure orientations as these are generally sub-paralleling the drillhole axis. Thus, these holes have little to contribute to domain definition as they are poorly oriented samples. Additionally, their location and orientation in space limits their usefulness if the design pit wall must be spatially adjusted. Basically, unless very carefully designed, they are wasted drillholes.

- Access may be an issue. In some areas, the rock face cannot be approached due to safety concerns; either imposed by regulation or by obvious danger. While structural spatial characteristics can, in most cases, be obtained utilising photogrammetric techniques in such areas, obtaining physical characteristics of the discontinuities can be somewhat challenging.

These are only a few of the myriad of biases that can accrue in the category of sample location bias when collecting structural spatial characteristic data. One must be cognisant of such concerns and address them as best one can as they arise. As structural domains are modified during iterative analysis, location bias will often require additional redress.

\subsection{Analytic bias}

Once sampling methodologies and locations are addressed, and data is collected, a third source of error/bias is encountered. This is analytic bias, or the bias accrued by the collected data as a function of the analytic methods chosen for data reduction. Some simplistic examples follow:

- Discontinuity orientations are often defined as 'sets' or clusters of discontinuity poles on a stereonet (Figure 1). These clusters are, in nature, a function of their inherent distributional forms as well as relative order of generation. As such, they may exhibit a variety of shapes, and overlap, on the common equatorial polar projection utilised for discontinuity set definition. They are decidedly not defined in nature as polar arc segments of dip/dip direction. In fact, dip and dip direction, while useful, are simply mathematical constructs to allow us to relate structure to a hypothetical horizontal surface. Yet, discontinuity sets are often defined by just such dip/dip direction hard bounds. This artificially constrains the data, potentially biasing not only the discontinuity set mean vectors, but the distributional range and shape as well. As can be seen in Figure 1, the discontinuity set confidence bounds are not constrained by any fixed dip or dip direction, but instead vary as a function of the discontinuity population in space.

- Clustering algorithms are occasionally utilised by some practitioners for discontinuity set determination. Yet, in the author's opinion, they fail in their task if the assumption of pole independence is violated; a common occurrence in some environments. Figure 1 demonstrates just such an example of overlapping discontinuity sets. Stereonet $A$ has been derived from major structure mapping with the set type differences determined from field mapping/interpretation, i.e. structural independence. Stereonet B was constructed from photogrammetric mapping with discontinuity set bounds derived from the assumption that structures in the overlap zone may belong to either discontinuity set (non-independence). Such discontinuity set interaction occurs frequently and, in many cases, the origin and nature of the overlap is critical to domain definition. It also, in some cases, has a decided effect on slope design given the potential dependence and interaction between the discontinuity sets.

- As was discussed earlier, drillhole discontinuity orientation data is orientation biased. The commonly utilised Terzaghi correction factor does not adequately compensate for the underlying orientation distribution, assuming independence between points, nor does it provide adequate (if any) orientation adjustment in the so-called 'blind zone' (Davy et al. 2006). Using this correction blindly can, and has, contributed to errors in domain determination. While it may seem difficult to believe, entire structural domains have been delineated based solely on drillhole orientation bias and/or correction and the failure to carefully adjust analytically, as best as one 
could, for said bias. Once the bias was addressed (in one case by additional data collection) different structural domains emerged.

- The methodology utilised to calculate discontinuity spacing (density) influences the value derived. For example, surface mapping from cell mapping derived spacing when utilising the orientation distribution and the mean vector for calculations will likely differ from a simple trigonometric correction on scanline mapping of the same area. So too will it likely differ from downhole set spacing (density) as obtained from drill core, again in the same area. This latter is especially true if spacing information is collected from barrel oriented discontinuities. As noted previously, orientation is often lost in fractured zones. This can heavily bias discontinuity set spacing upwards, producing what may be a non-conservative effect on a parameter affecting design.

- Discontinuity persistence by set can be unique to the methodology utilised for collection/calculation. One cannot simply conduct a bulk compilation of persistence by set and expect to calculate a reasonable trace length value for a discontinuity set (Figure 2). Trace lengths obtained by distribution free assessment methods will generally differ from the raw population of measured trace lengths obtained unless appropriate corrections are applied. Similarly, the effects of censoring on the data can be quite large and must be addressed.

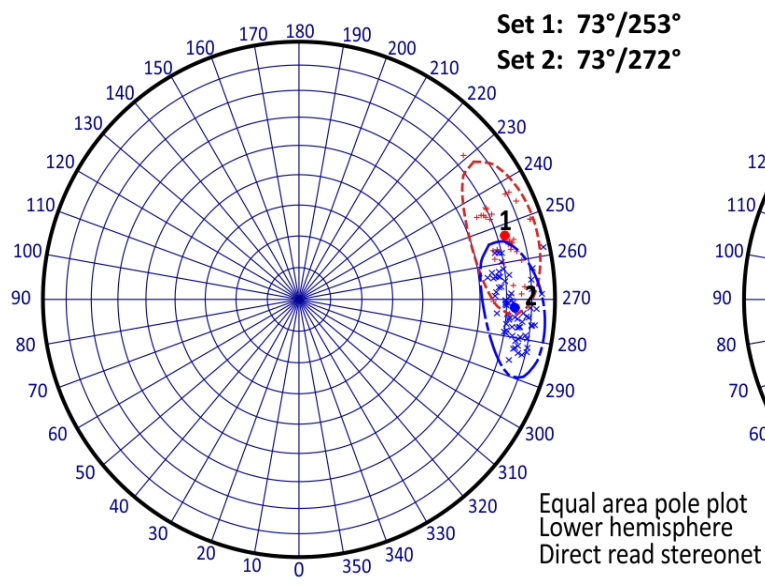

A

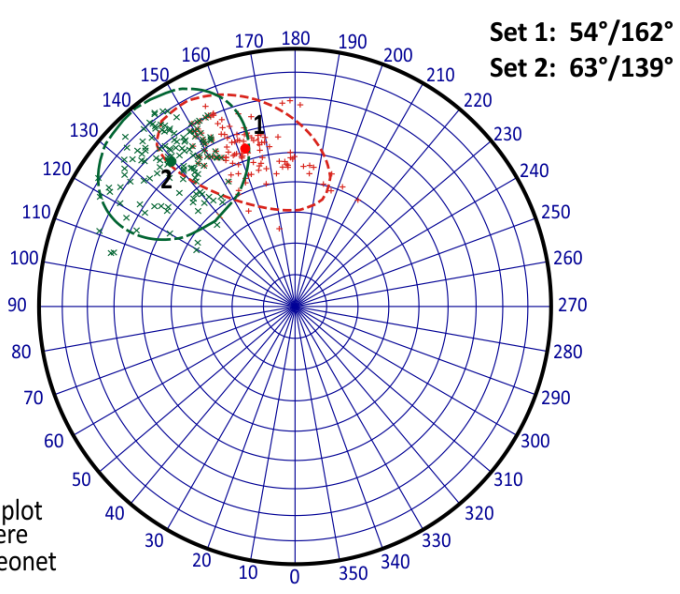

B

Figure 1 Overlapping discontinuity sets

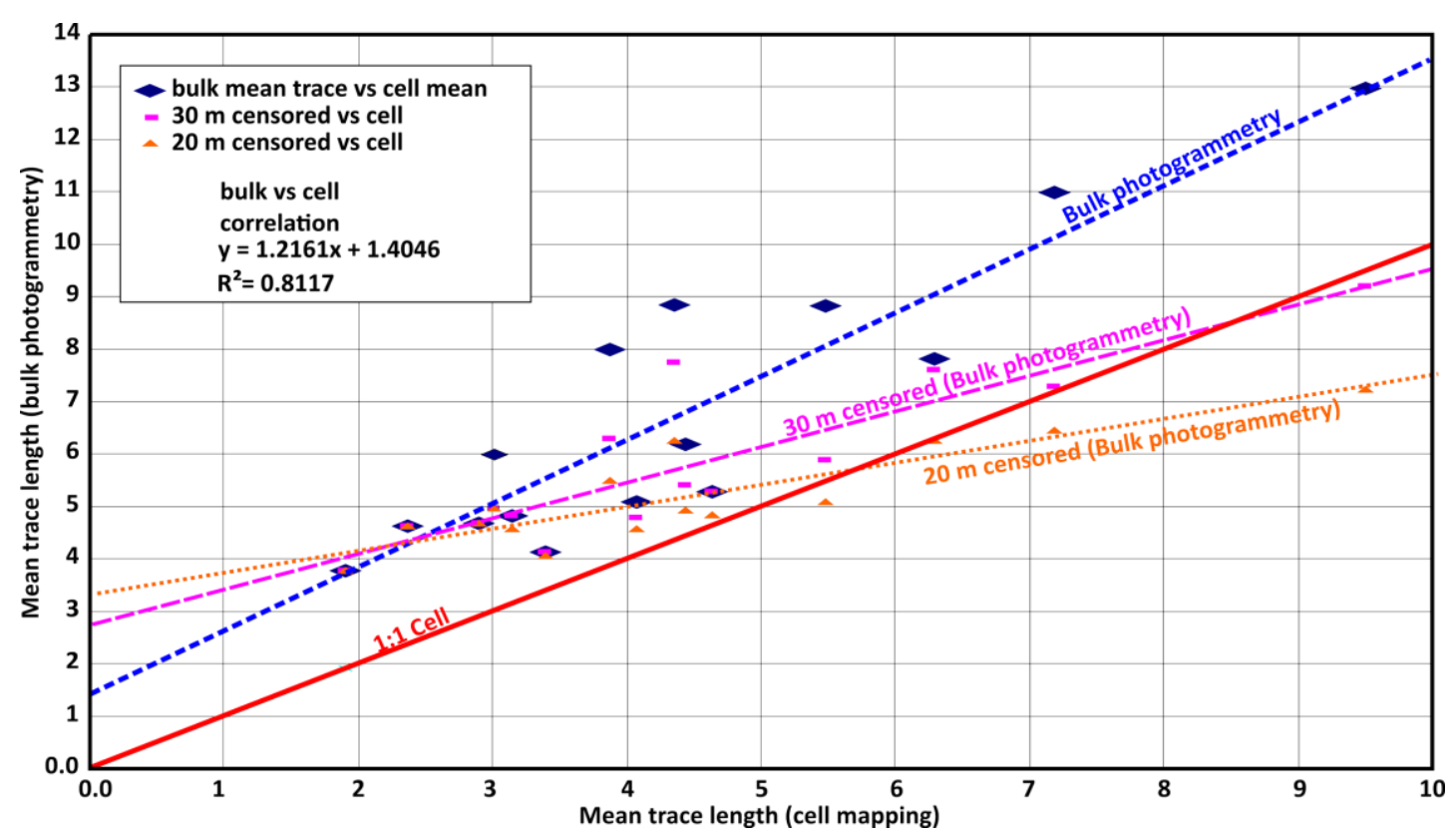

Figure 2 Analytic persistence bias (after Mathis 2014) 
Thus, when designing a data collection program, the analytical methods utilised to reduce, as well as combine, the data must be assessed for compatibility as well as minimise (as best possible) the analytic biases, not only for accurate structural domain determination, but for accurate stability analyses at a later date.

\section{Initial structural domain determination}

Structural domain determination techniques vary dependent on the data at hand. While efforts can be made to minimise the bias inflicted damage on the raw data, as discussed briefly in the previous sections, each and every project is unique.

The rock fabric domain determination method utilised by the author begins with simple polar stereonets of:

- Rock fabric - with all lithologies combined. However, each structural orientation observation is tagged with lithology, structure type, spatial location, and, if available, infill, roughness, persistence, terminations etc.

- Interpreted major structure (IMS) - with all lithologies combined. These are interpreted major structural, and associated, features from drill holes and from surface mapping. They maintain the same tags as for rock fabric with the potential inclusion of true thickness and comments regarding characteristics.

These two plots provide an overview of what the general structural trends may be on site. It can certainly be misleading, but the plot of IMS can be very useful in roughly visualising what the controlling bounds may be on the local structural domains. Of course, it can provide a 'shotgun' plot with no significant data concentrations. This is especially true of the fabric plot. However, even a result such as this is informative; potentially indicating the structural domains may be complicated and variable.

The exercise is then repeated for data sorted by lithology, structure type, lithology and alteration etc. This provides baseline information as to what discontinuity sets are typical and dominate, at least within the data set available.

There is no established procedure after this point, that the author is aware of, for further segregating data in an effort to define domains. A large part depends on how much and what type data is available, and the complexity of geologic structure, lithology and alteration.

The following general procedures for structural domain determination are normally utilised by the author:

- If at all possible, a preliminary IMS model is created. This consists of triangulated surfaces created from observations (drillholes or surface mapping) and existing (known) major geologic structure in the area of examination. Similarly, the lithology is plotted as triangulated surfaces. Both are, as noted, preliminary and generally change as domain definition proceeds.

- If there are limited amounts of fabric data, i.e. a small number of drillholes and surface outcrop, the individual drillholes/outcrop structure orientations are plotted on stereonets and then arranged spatially by collection locale. This includes the drillhole traces and the oriented sections of the drillhole, if the hole is not oriented in its entirety.

- If the area to be examined is of a depth such that the structural domains may vary by depth within the confines of the data, then elevation bounded 'levels' of data can be plotted in a similar fashion as for the two previous bullets. This is especially true if data from underground openings or long drillholes are incorporated in the data set.

- If sufficient data is available, the data can be divided into generally uniform blocks and evaluated in a somewhat similar fashion. These can be either two dimensional (plan only) or three dimensional (solids). If discontinuity sets are picked on such a data array it then allows various statistical methods to be utilised to discern orientation variation in the area of analysis. The IMS model is ignored for initial assessment, with the data re-evaluated as a function of the IMS bounds subsequent to initial evaluation. 
- Areas of similar structural set orientations are then selected through manipulation of the rock fabric orientation data, taking care to account for orientation and location bias in the collected data. At this point the IMS model is generally rebuilt in an effort to incorporate the structural orientation sets determined by the initial domain work. This will, of course, generally involve a reassessment of IMS discontinuity sets in light of the segregated fabric data. Additionally, the lithologic and alteration models are generally re-evaluated due to the potential changes imparted by variation of the throughgoing structural features.

- Rock fabric data is once again sorted within the preliminary structural domains, stereonets plotted, and discontinuity sets and associated spatial characteristics calculated. This is the first, and overview, structural domain assessment (Figure 3, statistics omitted in figure).

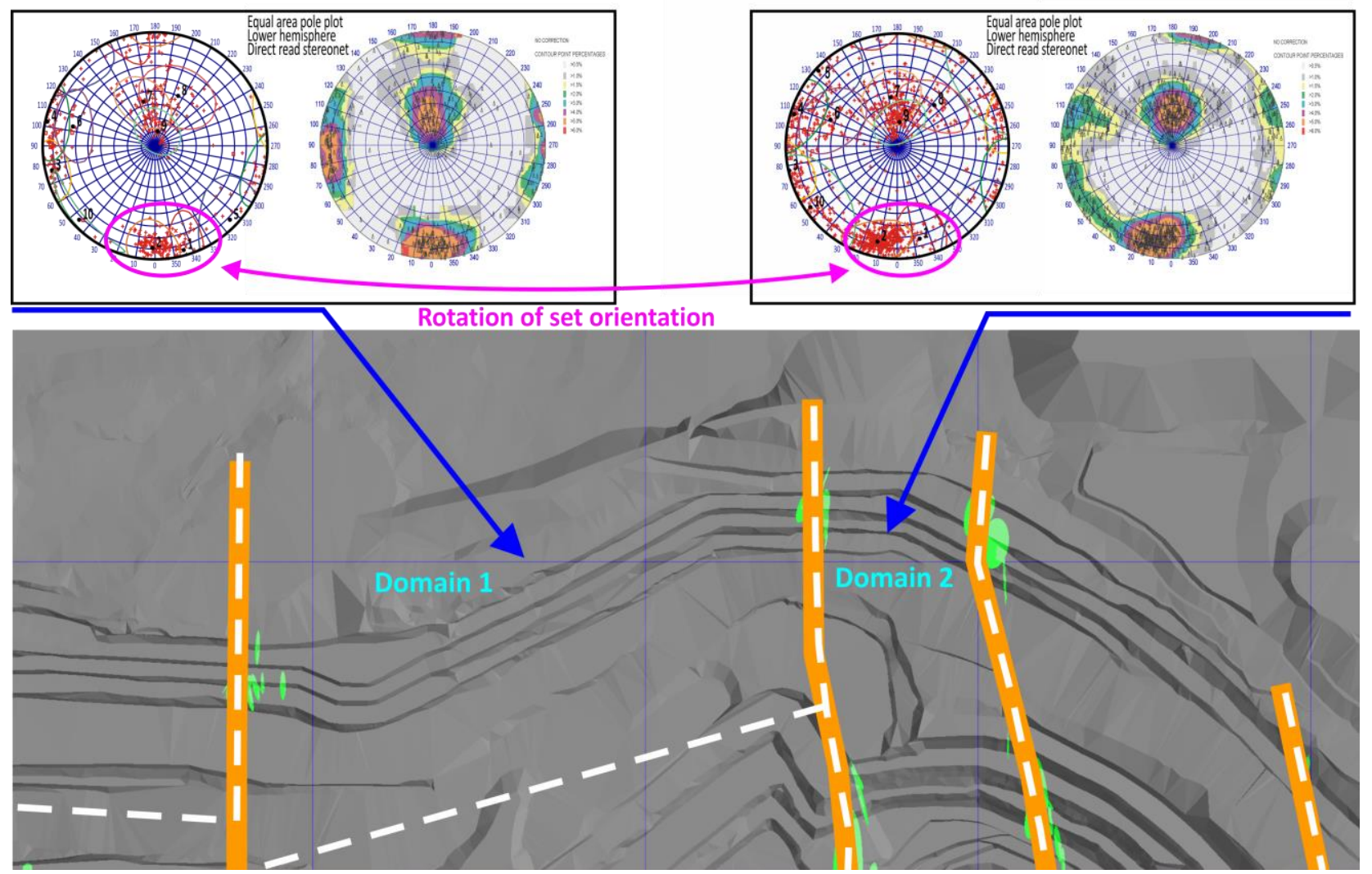

Figure 3 Generalised geotechnical structural domain definition (after Mathis 2011)

The preliminary rock fabric domain definition process is not complete at this point as only groupings by structural orientation have been assessed for rock fabric. The potential exists for internal systemic variation of local discontinuity orientation, persistence, spacing (density), infill etc.

Thus, the preliminary rock fabric structural domains are examined for such internal variations. For example, while general fabric orientations by set may be similar within the domain, discontinuity persistence and density may vary by area within the domain. A typical example is found near a fault, where structures paralleling the fault are both more frequent and exhibit greater persistence. Another example is near surface weathering/exfoliation, with a notable difference found in persistence/density as compared to depth. Still another is the effect of alteration on persistence/density, and/or infill characteristics, even if the local structural domain being examined is within the same lithologic unit.

Rock fabric is just one end of the spectrum in the determination of structural domains. For pit slopes, major structure is often a controlling factor for overall slopes and interramps. Proximity to a throughgoing regional fault or shear may result, in a fashion similar to that mentioned for fabric, in an increase in local faults proximal to the parent feature. These sub-parallel features can, at times, be evaluated as individuals. In other situations, the damage zones and associated sub-parallel faulting may become a structural domain, 
or even multiple domains. This is especially the case with multiple smaller fault defined blocks or alteration/fault motion contributing to significant local changes in the structural picture. At times, faults can, for lack of a better term, dissect an area sufficiently that the faulting controls the overall domain 'character'. The rock fabric may be substantially rotated within the local fault defined blocks but given the small size of these blocks it becomes impossible to utilise fabric as a structural domain defining characteristic.

Of course, the IMS structural domains can be imprinted over the rock fabric structural domains, creating a two tier situation for continued evaluation and design purposes.

\section{$4 \quad$ Continued structural domain evaluation}

At this point, one has a rough idea what structural domains may exist in the design area. However, the work necessary to convert these initial structural domains into design structural domains is far from over.

For example:

- There may be areas where structural definition is uncertain, even to the point of not knowing what is transpiring structurally or what controls the area. In this case, if possible, a hypothesis should be developed for the area based on the initial major structure and rock fabric domains. This will be necessary for additional evaluation.

- At times, it may not be possible to adequately define structural domain bounds given the available IMS and rock fabric data. Even though domainal differences may be obvious, the defining bounds are not.

- Some structural domains may have too little data to provide reliable structural estimates.

- Some structural domains may exhibit adverse characteristics critical to stability. If these are high risk areas, it may be necessary to increase the certainty regarding initially defined characteristics.

- Alternatively, structural domains may exhibit few, if any adverse characteristics. These are areas that may provide economic opportunities in design and, as such, must be accurately defined.

- Specific spatial characteristics may be lacking for some structural domains. For example, if a domain is defined solely by drilling, and persistence information is required for bench face angle (BFA) reliability assessments, how will this be determined?

- General discontinuity set characteristics may be observed, together with IMS orientations/relationships, which infer a specific tectonic origin. At times, this may be critical to design.

The above is an abbreviated list of situations that have been encountered by the author. The specific situation at hand, including the required information for design, guides the ongoing domain definition process.

What is of import to note here, and was implicit in the bullet points above, is that the excavation design begins to more strongly influence data collection and evaluation in this stage of domain evaluation. The engineer(s) conducting the structural domain segregation may begin to introduce the practicality of further domain definition, and thereby data collection, into the process. In other words, is what is available at this point good enough? What will be the impact of improved structure domain definition?

- In many cases, small but discernible changes in rock fabric domain characteristics will have limited, if any, practical effects on slope design. In some cases the design impact, especially on BFA reliability, is so small that it cannot even be observed when calculating BFAs around the pit. In this case, unless some fabric concern is critically important to interramp or overall stability, and if perceived accuracy is 'good enough', there may be no reason to collect additional fabric information in these domains. 
- 'Short' pit slopes (side hill cuts) are another obvious area for potentially saying the preliminary model is good enough on the downhill (short) side of the pit. If the slope is only to be a few benches in height, the overall impact of a conservative design to accommodate uncertainty in the structural domain characteristics may not be significant. It may even be less than the cost of the necessary additional data collection.

- Throughgoing 'fracture' zones (shattered faults) of significant width may also be an area where the initial domain definition is sufficient. Of course, this depends on the location and orientation relative to the design slope. However, if the fractured zone is steeply dipping, intersects the design slope at high angles, does not parallel and is not incorporated in benches, one may consider using the preliminary structural characteristics for such a feature as well. The benches will likely be irregular in this area but fabric definition may be extraordinarily difficult given the host environment. Again, a conservative estimate will likely be better than spending considerable money on drilling which may not increase accuracy or confidence.

Note that in each example given above consideration is given to the design impacts of the structural domain, whether it be rock fabric or IMS. The benefits, costs, and risks associated with not collecting additional information can drive the work conducted for continued structural domain evaluation.

One must not neglect what can be called the 'mini' or 'micro' structural domains, a terminology applied simply to distinguish domains that may be lost in general interpretation but could have an outsized practical impact on design.

- Faults that sub-parallel and are incorporated in a pit slope often belong to the 'mini' domain class, whether this be an IMS domain or a single feature of 2-5 $\mathrm{m}$ width. These are often examined as to overall and interramp slope stability but in many cases otherwise ignored. However, such features can be slope controlling due to their impact on the BFAs. Depending on their impact it may be well worth the time, effort, and money to accurately locate and characterise such features relative to the wall. It could be much more economic than a stepout to re-establish benches at a later date.

- Local discontinuity development immediately adjacent to a fault may only be 10-20 m in width, if that. However, the geologic structures within the zone may be distorted (folds, flares etc.) and exhibit extreme persistence. These need not be parallel to the slope to contribute to continuing local instability. Accurate characterisation of such zones allows the pit designer to modify wall orientations and locations to minimise the impact of such features. Whether domainal definition work is worth it or not is a function of the slope and the operation. If slight modifications can be incorporated in the original design it may very well be. If these zones are not somehow specifically flagged they will, in many cases, be lost during pit shell generation, potentially resulting in problems that could have been avoided.

There are also volumes of rock that defy simplistic description as structural domains, yet are obviously controlled by geologic structure.

Two such examples that come to mind in this regard can be found in folded terrain:

- Say, for example, an isoclinal fold, with the fold axis plunging obliquely along the axis of the pit. Theoretically the discontinuities will be rotating as a function of the location within the fold. However, there may also be curvilinear faults mimicking the fold. Both will likely impact local and overall slope design and both must be generally described such that they can be incorporated in said slope design. Here, in terms of practicality, one solution is to assume solids for the volumes that generally represent 'similar' fabric whereas one does the best that one can in interpreting the fold parallel faulting.

- Alternatively, the folded faults can be quite tight, with fold axes every $20-80 \mathrm{~m}$, and plunging into the pit. These can, especially with the fold parallel discontinuities/faults, control not only the 
benches but potentially the overall slope. Determining these accurately would require an inordinate amount of oriented core drilling, if even possible. Surface mapping may be the best method to even roughly describe the folding for geotechnical purposes. Even this may only be in a rough sense. For this particular case, the 'structural domain' will likely consist of curvilinear features approximated in terms of overall characteristics. Adjustments and characterisation may, and likely will, be required throughout the slope excavation process.

There are certainly other situations that can be envisioned, with many being a function of fault intersections, dissected fault/fabric and deformed/undulating surfaces.

\section{Conclusion}

Structural domains may be revised throughout the course of slope excavation. If it is a mine, the process is completed when mining is completed. That is, if the mine excavation is not re-purposed. If it is a civil excavation, the work may never be done. If problems, or changes, are encountered or required in the future, the structural domains may be revisited.

The definition of structural domains is a complex task, requiring thorough knowledge not only of structural geologic processes, but data collection methods and the biases associated with both data collection and data reduction. The designer must then have the ability to assimilate and process this information in such a fashion that reflects geotechnical reality in the project area sufficient for design purposes. All the while the designer must balance data collection costs, and possibilities, with the potential benefit of additional economic investments in the endeavour.

Such work requires the complete investment of senior engineers with substantial experience in the field, involving themselves every step of the way. Structural domain determination, and the necessary data collection for such determination, should not be simply relegated to junior engineers as the experience factor is enormous. And, as accurate structural domain interpretation can have a tremendous impact on pit slope angles (and associated risk reduction), it is an area where the geotechnical designer should consider substantial investment in thorough and accurate physical data collection and analysis, together with the aforementioned technical expertise.

\section{References}

Davy, P, Darcel, C, Bour, O, Munier, R, \& de Dreuzy, JR 2006, 'A note on the angular correction applied to fracture intensity profiles along drill core', Journal of Geophysical Research, vol. 111, November 2006, pp. B11408-B11414.

Mathis, JI 2011, 'Photogrammetric discontinuity mapping as applied to structural interpretation and drillhole planning at Barrick's Williams pit', Proceedings Slope Stability 2011: International Symposium on Rock Slope Stability in Open Pit Mining and Civil Engineering, Vancouver.

Mathis, JI 2014, 'To DFN or not to DFN - A question of circumstances', in Proceedings of DFNE2014, Vancouver.

Mathis, JI 2015, Geotechnical Data 1 - Rock Fabric and Structures, Edumine course, viewed May 2016, http://www.edumine.com/ courses/online-courses/geotechnical-data-1-rock-fabric-and-structures/ 